\title{
Podium
}
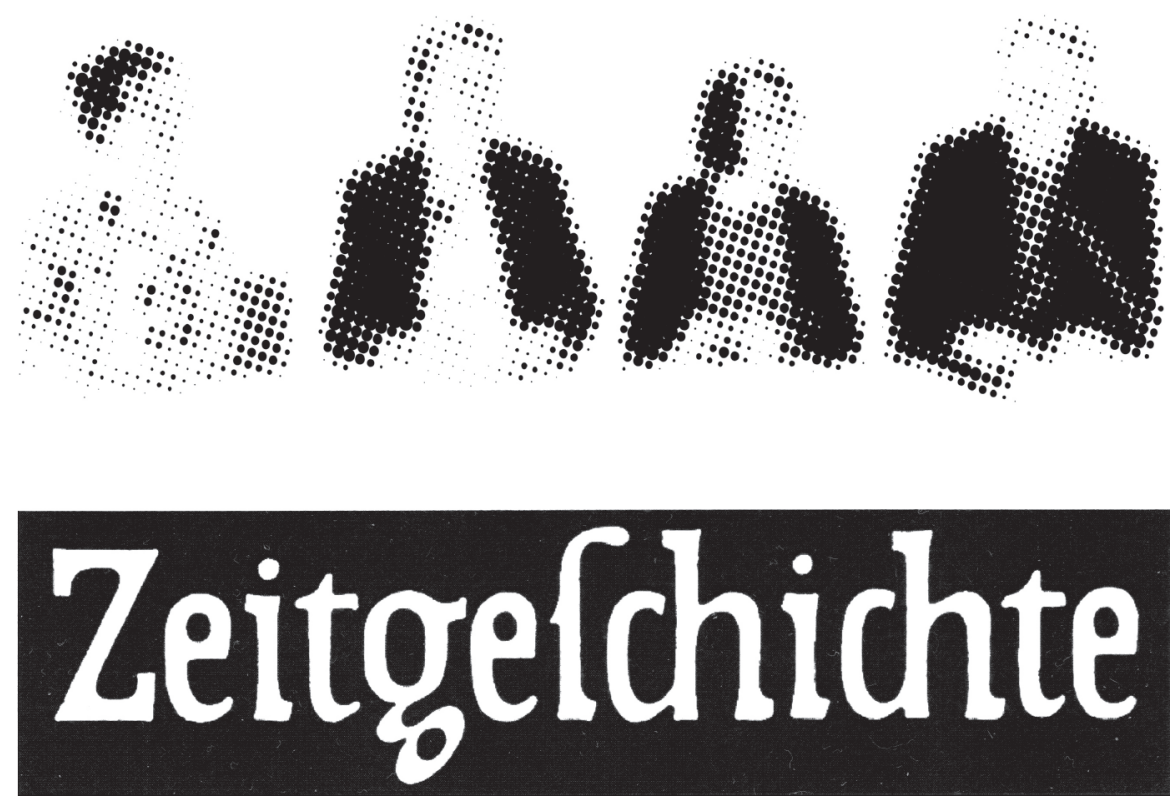
Podium Zeitgeschichte

\section{Wie nah ist uns die Zwischenkriegszeit?}

Geschichte und Aktualität der demokratischen Staatsgründungen nach dem Ersten Weltkrieg in Deutschland, Österreich, Polen, Litauen und der Tschechoslowakei

\section{Einführung}

Die spannungsreichen Jahre zwischen den beiden Weltkriegen des 20. Jahrhunderts werden im internationalen zeitgeschichtlichen Sprachgebrauch noch immer als die Zwischenkriegszeit bezeichnet (the Interwar Period oder the Interwar Years, l'entre-deux-guerres etc.). Die Kohärenz der Epoche ist dabei nicht vorgegeben; vielmehr resultiert sie in der Regel aus der gewählten geschichtswissenschaftlichen Perspektive. Ein möglicher Zugang liegt etwa in der Betrachtung des sich neu strukturierenden europäischen Staatensystems. Ein anderer, für die europäische Geschichte der Zwischenkriegszeit fundamentaler Ansatz zielt auf die Formierung und innere Verfasstheit von Staaten: Die Zwischenkriegszeit war eine Phase der staatlichen Neugründung, des demokratischen Aufbruchs, aber auch der Krisen und des Scheiterns von Demokratien sowie der Etablierung autoritärer und diktatorialer Systeme.

Nachdem die Epoche zwischen den Weltkriegen zeitweise ein wenig in den Hintergrund des wissenschaftlichen und öffentlichen Interesses getreten war, scheint sie uns seit einigen Jahren wieder näher gerückt zu sein. Demokratiegeschichtliche Fragen spielen hierfür eine maßgebliche Rolle: Die gewachsene mediale und wissenschaftliche Präsenz der Zwischenkriegszeit ${ }^{1}$ hängt zum einen ganz banal - mit der Wirkmächtigkeit runder Jahreszahlen zusammen. Der demokratische Aufbruch, den das Deutsche Reich sowie verschiedene Staaten aus der Konkursmasse der Donaumonarchie und des Zarenreichs mit oder nach dem Kriegsende von 1918 vollzogen haben, jährt sich nun zum hundertsten Male. Zum anderen kommt ein Faktor ins Spiel, der mit den Problemen unserer Gegenwart zu tun hat. Die Krisenerscheinungen, die demokratische Systeme derzeit zeigen - insbesondere im östlichen Europa -, und die neue Attraktivität rechtspopulistischer Strömungen und autoritärer Systeme lassen sich als Aufforderung verstehen, dem Schicksal demokratischer Staaten in der Zwischenkriegszeit mehr Interesse zu widmen.

Vor diesem Hintergrund hat das von Johannes Hürter, Thomas Raithel, Thomas Schlemmer und Jürgen Zarusky konzipierte zweite Podium Zeitgeschichte der

\footnotetext{
${ }^{1}$ Vgl. Boris Barth, Europa nach dem Großen Krieg. Die Krise der Demokratie in der Zwischenkriegszeit 1918-1938, Frankfurt a. M./New York 2016; Steffen Kailitz (Hrsg.), Nach dem „Großen Krieg“. Vom Triumph zum Desaster der Demokratie 1918/19 bis 1939, Göttingen 2017, und Erwin Oberländer (Hrsg.), Autoritäre Regime in Ostmittel- und Südosteuropa 1919-1944, 2., um ein Nachwort ergänzte Aufl., Paderborn 2017.
} 
VfZ dazu eingeladen, Geschichte und Aktualität der demokratischen Staatsgründungen nach dem Ersten Weltkrieg an verschiedenen Beispielen zu diskutieren und die in der politisch-historischen Diskussion der Bundesrepublik klassische Frage nach der Nähe der Zwischenkriegszeit in einen komparatistischen Rahmen zu stellen. Dies geschieht im Folgenden durch fünf thesenhafte Beiträge, die sich mit Deutschland, Österreich, Polen, Litauen und der Tschechoslowakei beziehungsweise der heutigen Tschechischen Republik beschäftigen. Hier, im mittelund osteuropäischen Raum, sind 1918/19 eine Reihe neuer Staaten entstanden, hier wurde auf breiter Front im Bereich mehr oder weniger konstitutioneller Monarchien ein demokratisches und republikanisches Staatsmodell etabliert und hier war - sieht man von der bis 1938 demokratischen Tschechoslowakei ab - die Demokratie teils sehr schnell, teils in der ersten Hälfte der 1930er Jahre gescheitert. Mit Ota Konrád (Karls-Universität Prag), Ekaterina Makhotina (Rheinische Friedrich-Wilhelms-Universität Bonn), Anton Pelinka (Central European University Budapest) und Krzysztof Ruchniewicz (Willy Brandt Zentrum der Universität Wrocław / Breslau) melden sich - zusammen mit Thomas Raithel aus dem Institut für Zeitgeschichte - ausgewiesene Spezialisten zu Wort.

Die Autorin und die Autoren, die in der Anlage ihrer Beiträge weitgehend frei waren, wurden gebeten, drei Leitfragen zu berücksichtigen:

1. Von welcher Position aus erfolgt heute der Rückblick auf die Zwischenkriegszeit?

2. Wie lässt sich aus aktueller wissenschaftlicher Sicht die Geschichte der jeweils betrachteten Demokratie in der Zwischenkriegszeit beurteilen?

3. Welche Relevanz hat die nationale Demokratiegeschichte der Zwischenkriegszeit für die Gegenwart?

Dass die Autorin und die Autoren in ihren Beiträgen ganz unterschiedliche Schwerpunkte gesetzt haben, vermag angesichts stark voneinander abweichender historischer Kontexte und spezifischer nationaler Traumatisierungen kaum zu überraschen. Europäische Geschichtsschreibung des 20. Jahrhunderts hat es in hohem Maße mit nationalgeschichtlichen Entwicklungen und Prägungen zu tun, deren komparatistische sowie beziehungs- und transfergeschichtliche Zusammenführung trotz mancher Bemühungen in den letzten Jahrzehnten erst in den Anfängen steckt.

In der Tschechoslowakei, in Litauen, Polen und Österreich, wo es jeweils noch keine gefestigten Nationalstaaten gab, war das Problem der eigenen Staatlichkeit und Unabhängigkeit beziehungsweise der nationalen Identität fundamental für die innere Entwicklung der Zwischenkriegszeit und damit auch für das Schicksal der Demokratie. Die vorliegenden Beiträge machen dies sehr deutlich. In den drei erstgenannten Fällen erscheint das Problem auch gegenwärtig noch präsent: In der 1993 durch die Trennung von der Slowakei entstandenen tschechischen Republik zeigt es sich - eher subtil - im verbreiteten Bekenntnis zur einstigen, ganz anders zugeschnittenen Tschechoslowakischen Republik. In dem seit 1990 unabhängigen Litauen ist die Frage der Staatlichkeit und nationalen Identität hingegen weiterhin virulent: Hier dient nicht die demokratische Episode bis Mitte der 1920er Jahre als affirmativer historischer Bezug auf die Zwischenkriegszeit, 
sondern die 1926 durch einen Militärputsch begründete Diktatur von Antanas Smetona sowie die damit verbundene Konstruktion wirksamer nationalgeschichtlicher Narrative. Die seit 1989 bestehende Dritte Polnische Republik - schon der Name ist ein Brückenschlag zur Zweiten Republik der Zwischenkriegszeit - erlebt derzeit nach einer intensiven Phase der politischen und soziökonomischen Transformation den massiven Bedeutungsgewinn nationalistischer und auf einen „starken Staat“ zielender Diskurse. Auch hier richtet sich die historische Bezugnahme - zumindest seit der erneuten Regierungsübernahme der PiS-Partei (Prawo i Sprawiedliwość; auf Deutsch: Recht und Gerechtigkeit) im Jahr 2015 - kaum noch auf die demokratischen Jahre der Zwischenkriegszeit. Ähnlich wie in Litauen steht ein autokratischer Herrscher im Zentrum der von Regierungsseite propagierten Erinnerungskultur: Józef Piłsudski, dessen Staatstreich 1926 die demokratische Phase der Zweiten Polnischen Republik beendet hat. In der 1945 begründeten Zweiten Republik Österreichs scheint die Frage der eigenständigen, von Deutschland separierten nationalen Identität schon lange geklärt - auch in einer bewussten Absetzung von der Ersten Republik, die für zentrale Bereiche des politischen Lebens charakteristisch war und ist.

Ein anderes Bild ergibt sich für Deutschland: In keinem der hier behandelten Staaten scheint gegenwärtig der Bezug auf die Demokratieerfahrungen der Zwischenkriegszeit so intensiv. Hier manifestiert sich der überragende Stellenwert, den die Geschichte der gescheiterten Weimarer Republik für die politische Kultur der Bundesrepublik und ihr (vielfach kontrastives) Selbstverständnis gewonnen hat. Die jahrzehntelange demokratiegeschichtliche „Aufarbeitung“ - die weder kohärent noch abgeschlossen ist - stellt im Vergleichsrahmen unseres Podiums weitgehend eine deutsche Besonderheit dar.

Dass dies eine unabdingbare Folge der deutschen NS-Geschichte ist, braucht hier nicht dargelegt zu werden. Völlig abweichend war die Situation nach 1945 in den anderen hier behandelten Staaten. Während in Österreich eine erfolgreiche Neubegründung der Demokratie stattfand und gleichzeitig die NS-Zeit weitgehend von der neuformierten eigenen nationalen Identität getrennt wurde, hatte die Demokratie in Polen, in der Tschechoslowakei und im baltischen Raum, der zudem Teil der UdSSR blieb, keine Chance. Dies gilt auch für eine wie auch immer geartete „Aufarbeitung“ demokratischer Erfahrungen aus der Zwischenkriegszeit. Als sich dann nach 1989 Freiräume geboten hätten, stand die dringlichere Auseinandersetzung mit der kommunistischen und fremdbestimmten Vergangenheit der Epoche nach dem Zweiten Weltkrieg im Vordergrund; zudem waren die politischen Akteure der Zwischenkriegszeit meist nicht mehr am Leben.

Auffallend ist, dass in den Beiträgen zur Tschechischen Republik und zu Polen die kritische Beschäftigung mit dem demokratischen Erbe der Zwischenkriegszeit als programmatische Perspektive auftaucht, die helfen könnte, Simplifizierungen und Defizite des verbreiteten Geschichtsbildes zu überwinden. Im tschechischen Fall bezieht sich dies auf die im populären Geschichtsbewusstsein seit 1989 erkennbare Idealisierung der Tschechoslowakischen Republik und auf das Postulat, diese in ihrer Widersprüchlichkeit sowie in ihren inter- und transnationalen Kon- 
texten zu analysieren. Im Falle Polens geht es grundsätzlicher um eine Überwindung des demokratiegeschichtlichen Desinteresses gegenüber der Zweiten Polnischen Republik.

Wie nah ist uns die Zwischenkriegszeit? Die Antwort auf diese Frage hängt immer davon ab, was man unter Nähe versteht. Bemessen am Grad der öffentlichen Diskussion ist eine Nähe wohl am ehesten im deutschen Falle erkennbar, und gerade in den letzten Jahren hat sie wieder zugenommen. Dies schließt die antithetische Abgrenzung ein, wenngleich sie zu einem selbstgefälligen Schematismus werden kann. Für die Bundesrepublik Deutschland sind diese Antithetik und das damit verbundene Bemühen, historische Lehren zu definieren, seit langem charakteristisch. Diese Haltung hat wohl auch die mediale Fixierung eines stets präsenten pauschal-negativen Weimarbildes gefördert, über das die Geschichtswissenschaft schon längst hinaus ist.

Bewertet man hingegen die Analogie historischer Konstellationen und das Anknüpfen an vergangene Formen des Staats- und Gesellschaftsverständnisses als Nähe, dann zeigt sich im heutigen Deutschland eher eine große Ferne von „Weimarer Verhältnissen“ - was zweifellos auch mit der erfolgreichen eigenen Demokratiegeschichte zu tun hat. Nahe an der Zwischenkriegszeit liegen in diesem Sinne vor allem Polen und Litauen. Allerdings bezieht sich dort die Nähe, wie erwähnt, nicht auf die demokratischen Ansätze, sondern auf das nachfolgende autoritäre System, das in den Verunsicherungen der Gegenwart ein attraktives Modell vorgibt.

Der Ansatz unseres Podiums ließe sich durch weitere europäische Perspektiven ergänzen: So wären die öffentlichen und wissenschaftlichen Debatten, die in Frankreich und Großbritannien über die nationale demokratische Entwicklung der Zwischenkriegszeit geführt werden, ebenfalls ein komparatistisch instruktives Thema. 1918 fand in Frankreich und Großbritannien nicht die Gründung eines demokratischen Staates statt, sondern die (zeitweise) Bestärkung des bestehenden Staates infolge des Erfolgs im Ersten Weltkrieg. Auch deshalb war das Prekäre der Demokratie in der Zwischenkriegszeit weniger ausgeprägt als in den hier behandelten Staaten. Inwieweit der Blick zurück in die Zwischenkriegszeit dadurch in Frankreich und Großbritannien insgesamt eine andere Ausrichtung gewinnt, wäre zu diskutieren.

Derart weiterführende Aspekte sollen, ebenso wie die in den Beiträgen angesprochenen Themen und Fragen, in einer im Institut für Zeitgeschichte stattfindenden und dann auf der Homepage dokumentierten Podiumsdiskussion (17. Mai 2018) behandelt werden. Auch das online zugängliche VfZ-Forum (www.ifzmuenchen.de/vierteljahrshefte/forum/) bietet hierzu Gelegenheit.

Thomas Raithel 